\title{
ON A CLASS OF DOUBLY TRANSITIVE GROUPS ${ }^{1}$
}

\section{J. L. ZEMMER ${ }^{2}$}

The purpose of this note is to prove the following.

TheOREM. Let $G$ be a group of permutations on a set $\mathfrak{M}$. If (i) $G$ is doubly transitive and only the identity fixes two letters, and (ii) the subgroup fixing one letter is Abelian, then $G$ is isomorphic to the group of affine transformations $x \rightarrow a x+b, a \neq 0$, on $a$ field.

This theorem is related to a result of Hall [2, Theorem 5.6], which states that if a group $G$ satisfies condition (i) above and in addition either

(i') $\mathfrak{M}$ is finite, or

(i") for some $i, j \in \mathfrak{M}$ there is at most one element of $G$ mapping $i$ into $j$ which displaces all of the letters, then $G$ is isomorphic to the group of affine transformations, $x \rightarrow a x+b, a \neq 0$, on a near-field. A near-field is an algebraic system $(K,+, \cdot)$ consisting of a set $K$ and two binary operations + and $\cdot$ satisfying:

(a) $K(+)$ is an Abelian group with identity 0 ,

(b) the nonzero elements of $K$ form a group with respect to $\cdot$ with identity 1 ,

(c) $x(y+z)=x y+x z$ for $x, y, z \in K$,

(d) $0 \cdot a=0$ for each $a \in K$,

(e) if $a, b, c \in K, a \neq b$, the equation $a u=b u+c$, has a unique solution $u$ in $K$.

In [1], Gorenstein has called an independent $A B A$ group, any group $H$ which contains two subgroups $A$ and $B$ such that for $x \in H$, either $x \in A$, or $x$ can be represented uniquely in the form $a_{1} b a_{2}, a_{1}, a_{2} \in A$, $1 \neq b \in B$. The proof of the Theorem will consist of first showing that a doubly transitive group $G$, in which only the identity fixes two letters, is a special kind of independent $A B A$ group. This is a corollary of Lemma 2. Using the structure of $G$ as an independent $A B A$ group, it will then be shown that, when $A$ is Abelian, $G$ satisfies condition $\left(i^{\prime \prime}\right)$ of Hall's theorem, from which our theorem follows at once.

It should be pointed out that in the finite case Hall's result [2, Theorem 5.6] follows almost immediately from the corollary of

Received by the editors April 21, 1960 and, in revised form, August 8, 1960.

1 This work was supported by the National Science Foundation.

2 The author is indebted to the referee for several suggestions, in addition to his pointing out the validity of Lemma 2 . 
Lemma 2 together with a result of Gorenstein [1, Theorem 6].

The original version of this paper began with the Corollary to Lemma 2. The referee has suggested a more general lemma relating doubly transitive groups to certain kinds of $A B A$ groups. This is stated as Lemma 2 . To state Lemma 2 it is necessary first to generalize the notion of independent $A B A$ group. This will be done with the aid of Lemma 1.

A group $G$ with subgroups $A$ and $B$ is called an $A B A$ group if for $x \in G, x=a_{1} b a_{2}$, where $a_{1}, a_{2} \in A, b \in B$. If $G$ is an $A B A$ group, and $b \in B, b \neq e$, the identity, define $L(G)$ and $R(G)$ as follows:

$$
\begin{aligned}
& L(G)=\{x \in A \mid b=x b y \text { for some } y \in A\}, \\
& R(G)=\{x \in A \mid b=y b x \text { for some } y \in A\} .
\end{aligned}
$$

Lemma 1. If $G$ is an $A B A$ group with $B$ of order two, then $L(G)$ $=R(G)$ is a subgroup of $A$, say $A^{\prime}$. Further, if $A^{\prime}$ is normal in $A$ then $A^{\prime}$ is normal in $G$.

Proof. Let $x \in L(G)$, and choose $y$ so that $b=x b y$. Then $b=b^{-1}$ $=(x b y)^{-1}=y^{-1} b x^{-1}$, hence $y b x=b$ and $x \in R(G)$. Thus, $L(G) \subseteq R(G)$. A slight modification of this argument shows that $R(G) \subseteq L(G)$; it follows that $L(G)=R(G)$. Again, let $x \in A^{\prime}=L(G)=R(G)$; then $b=x b y=y^{-1} b x^{-1}$, which implies $x^{-1} \in A^{\prime}$. If $x_{1}, x_{2} \in A^{\prime}$ then $b=x_{1} b y_{1}$, $b=x_{2} b y_{2}$. Clearly $b=x_{1}\left(x_{2} b y_{2}\right) y_{1}=x_{1} x_{2} b y_{2} y_{1}$, and $A^{\prime}$ is a subgroup of $A$.

If $A^{\prime}$ is a normal subgroup of $A$, and $x \in A^{\prime}, g \in G$, then $g^{-1} x g$ $=a_{2}^{-1} b a_{1}^{-1} x a_{1} b a_{2}=a_{2}^{-1} b x_{1} b a_{2}$, where $x_{1} \in A^{\prime}$. Since $b=x_{1} b y, b x_{1} b=y^{-1}$ $\in A^{\prime}$. Hence $g^{-1} x g=a_{2}^{-1} y^{-1} a_{2} \in A^{\prime}$. Thus $A^{\prime}$ is normal in $G$.

The following definition is a generalization of an independent $A B A$ group for the special case where $B$ has order two. An $A B A$ group $G$, where $B$ has order two, is called an $n$-independent $A B A$ group if $n$ is the order of the subgroup $A^{\prime}$, described in Lemma 1.

LEMMA 2. If $G$ is a doubly transitive group with subgroup fixing two letters finite, of order $n$, then $G$ is an $n$-independent $A B A$ group.

Proof. Let $G$ be a group satisfying the hypotheses of the lemma. Denote by 0 and 1 a pair of distinct letters of the set on which $G$ acts. Let $A$ be the subgroup of $G$ which fixes 0 , and $A^{\prime}$ the subgroup of $A$ which fixes 0 and 1 . If $c$ is an element of $G$ which interchanges 0 and 1 , then the subgroup, $\left\{A^{\prime}, c\right\}$, of $G$ generated by $A^{\prime}$ and $c$ is finite of order $2 n$. Thus, $\left\{A^{\prime}, c\right\}$ contains an element of order two. 'The double transitivity of $G$ implies the existence of an element $b$, of order two, which interchanges 0 and 1.

Now, let $g \in G, g(0)=\alpha, g(1)=\beta$, then $\alpha \neq \beta$. If $\alpha=0$, then $g \in A$; 
if $\alpha \neq 0$, let $a_{1}$ be an element of $A$ such that $a_{1}(1)=\alpha$. Since $a_{1}(1)$ $=\alpha \neq \beta$, it follows that $a_{1}^{-1}(\beta) \neq 1$, and hence $b a_{1}^{-1}(\beta)=\eta \neq 0$. Let $a_{2}^{*}$ be an element of $A$ such that $a_{2}^{*}(1)=\eta=b a_{1}^{-1}(\beta)$; then $a_{1} b a_{2}^{*}(1)=\beta$, and $a_{1} b a_{2}(0)=a_{1} b(0)=a_{1}(1)=\alpha$. It follows that $\left(a_{1} b a_{2}^{*}\right)^{-1} g$ fixes both 0 and 1 , hence $\left(a_{1} b a_{2}^{*}\right)^{-1} g=a \in A^{\prime}$, and $g=a_{1} b a_{2}^{*} a=a_{1} b a_{2}$. Thus $G$ is an $A B A$ group.

With the notation of Lemma 1 , let $x \in L(G) \subseteq A$, so that $b=x b y$ for some $y$ in $A$. Since $x \in A, x(0)=0$, and since $x=b y^{-1} b, x(1)$ $=b y^{-1} b(1)=b y^{-1}(0)=b(0)=1$. Thus $x$ fixes 1 as well as 0 and $x \in A^{\prime}$, whence $L(G) \subseteq A^{\prime}$. Conversely, let $a \in A^{\prime}$; then $b a^{-1} b$ fixes 0 and 1 . Thus, $b a^{-1} b=a^{\prime} \in A^{\prime}$, and $b=a b a^{\prime}$, and $a \in L(G)$. It follows that $A^{\prime} \subseteq L(G)$. Thus $L(G)=A^{\prime}$ has order $n$, and it is seen that $G$ is an $n$-independent $A B A$ group.

The converse of Lemma 2 is false. Consider, for example, the noncyclic group of order ten. It is a 5 -independent $A B A$ group and is not isomorphic to any doubly transitive group. Several modifications of the converse are true, and a particular one is proved in the following.

Corollary. A group $G$ is doubly transitive, with only the identity fixing two letters if and only if $G$ is an independent $A B A$ group with $B$ of order two.

Proof. Let $G$ be a doubly transitive group in which only the identity fixes two letters. It follows from Lemma 2 that $G$ is a 1 -independent $A B A$ group, that is, an independent $A B A$ group with $B$ of order two.

Conversely, let $G$ be an ABA group of this type, and $\mathfrak{M}$ the set of right cosets of $A$ in $G$. Each $g \in G$ determines a permutation $T_{g}$ on $\mathfrak{M}$, namely the mapping $A x \rightarrow A x g$. The set of mappings $\left\{T_{g}, g \in G\right\}$ forms a group of permutations on $\mathfrak{M}$, and it is readily seen that the mapping $g \rightarrow T_{\theta}$ is an isomorphism of $G$ onto this permutation group. To see that this group is doubly transitive, let $A x_{1} \neq A x_{2}, A z_{1} \neq A z_{2}$ be any two pairs of left cosets. Since $x_{2} x_{1}^{-1}, z_{2} z_{1}^{-1} \in A$, we have $x_{2} x_{1}^{-1}=a^{\prime} b a^{\prime \prime}$, $z_{2} z_{1}^{-1}=\bar{a} b \hat{a}$. Let

$$
y=x_{1}^{-1} a^{\prime \prime-1} \hat{d} z_{1}
$$

then

$$
A x_{1} y=A x_{1} x_{1}^{-1} a^{\prime \prime-1} a z_{1}=A z_{1}
$$

and

$$
A x_{2} y=A x_{2} x_{1}^{-1} a^{\prime \prime-1} \hat{a} z_{1}=A a^{\prime} b a^{\prime \prime} a^{\prime \prime-1} \hat{a} z_{1}=A b \hat{a} z_{1}=A b b \bar{a}^{-1} z_{2}=A z_{2} .
$$

Finally, to see that no element, other than the identity, has more 
than one fixed point, let $A x_{1} \neq A x_{2}$ and suppose that for some $y \in G$, $A x_{1} y=A x_{1}$ and $A x_{2} y=A x_{2}$. Then $x_{1} y=a_{1} x_{1}, x_{2} y=a_{2} x_{2}$, where $a_{1}, a_{2} \in A$. It follows that $y=x_{1}^{-1} a_{1} x_{1}=x_{2}^{-1} a_{2} x_{2}$, or that

$$
a_{1} x_{1} x_{2}^{-1}=x_{1} x_{2}^{-1} a_{2} .
$$

But, $x_{1} x_{2}^{-1} \notin A$, so that $x_{1} x_{2}^{-1}=\tilde{a} b \ddot{a}$. Thus,

$$
a_{1} \tilde{a} b \check{a}=\tilde{a} b \check{a} a_{2},
$$

which implies $a_{1} \tilde{a}=\tilde{a}, a_{1}=e, x_{1} y=x_{1}, y=e$. Thus $G$ is isomorphic to a doubly transitive group in which only the identity fixes two letters.

Before proceeding to the next two lemmas, we list some lemmas of Hall which will be needed. In [2] Hall proves that in a doubly transitive permutation group with only the identity fixing two letters, the following hold:

I. There exists one and only one element of order 2 which interchanges a given pair of elements of $\mathfrak{M}$.

II. The elements of order 2 are in a single conjugate class.

Two cases arise from II:

CASE 1. The elements of order 2 displace all elements of $\mathfrak{M}$.

CASE 2. Every element of order 2 fixes an element of $\mathfrak{M}$.

III. In Case 2 there is one and only one element of order 2 with a given fixed point.

IV. If $b_{1}, b_{2}$ are distinct elements of order 2 then $b_{1} b_{2}$ displaces all elements of $\mathfrak{M}$.

In terms of its representation as an $A B A$ group we see that in Case 1, $A$ contains no element of order 2; and in Case 2, $A$ contains a unique element of order 2 , which will be denoted by $t$.

With the notation of Lemma 2 , let $A^{*}$ be the set of nonidentity elements of $A$, and let $a \in A^{*}$. Then $b a b \notin A$ and hence $b a b=\phi(a) b \psi(a)$, where $\phi(a), \psi(a) \in A$. Further, $\phi(a) \neq e \neq \psi(a)$, so that $\phi, \psi$ are mappings of $A^{*}$ into $A^{*}$. Also since $b$ has order $2, b a^{-1} b=(b a b)^{-1}$, whence, $\phi\left(a^{-1}\right) b \psi\left(a^{-1}\right)=[\phi(a) b \psi(a)]^{-1}=[\psi(a)]^{-1} b[\phi(a)]^{-1}$. From the uniqueness of the representation it follows that

$$
\phi\left(a^{-1}\right)=[\psi(a)]^{-1} .
$$

In the following two lemmas it is assumed that the subgroup $A$ is Abelian. The element $t_{0}$ of $A$ will be the identity, $e$, in Case 1 and the unique element, $t$, of order two in Case 2.

Lemma 3. If $a \in A, a \neq t_{0}$ then $\psi\left(t_{0} a\right) \cdot \phi\left(t_{0} a\right)=a$.

Proof. First, from $b a b=\phi(a) b \psi(a)$, we obtain $a b=b \phi(a) b \psi(a)$ $=\phi^{2}(a) b \psi(\phi(a)) \psi(a)$, whence, $\phi^{2}(a)=a$, and 


$$
\psi(\phi(a)) \cdot \psi(a)=e .
$$

Similarly, $\psi^{2}(a)=a$ and it follows that $\phi, \psi$ are 1-1 mappings of $A^{*}$ onto $A^{*}$. From $b a_{1} b=\phi\left(a_{1}\right) b \psi\left(a_{1}\right)$ and $b a_{2} b=\phi\left(a_{2}\right) b \psi\left(a_{2}\right)$, we obtain,

$$
\begin{aligned}
b a_{1} a_{2} b & =\phi\left(a_{1}\right) b \psi\left(a_{1}\right) \phi\left(a_{2}\right) b \psi\left(a_{2}\right) \\
& =\phi\left(a_{1}\right) \phi\left[\psi\left(a_{1}\right) \phi\left(a_{2}\right)\right] b \psi\left[\psi\left(a_{1}\right) \phi\left(a_{2}\right)\right] \psi\left(a_{2}\right) .
\end{aligned}
$$

Also, $b a_{1} a_{2} b=\phi\left(a_{1} a_{2}\right) b \psi\left(a_{1} a_{2}\right)$, whence,

$$
\begin{aligned}
& \phi\left(a_{1} a_{2}\right)=\phi\left(a_{1}\right) \phi\left[\psi\left(a_{1}\right) \phi\left(a_{2}\right)\right], \\
& \psi\left(a_{1} a_{2}\right)=\psi\left[\psi\left(a_{1}\right) \phi\left(a_{2}\right)\right] \cdot \psi\left(a_{2}\right) .
\end{aligned}
$$

Since $A$ is Abelian we may interchange $a_{1}$ and $a_{2}$ in the right-hand sides of (3) and (4) to obtain

$$
\begin{aligned}
& \phi\left(a_{1} a_{2}\right)=\phi\left(a_{2}\right) \cdot \phi\left[\psi\left(a_{2}\right) \cdot \phi\left(a_{1}\right)\right], \\
& \psi\left(a_{1} a_{2}\right)=\psi\left[\psi\left(a_{2}\right) \cdot \phi\left(a_{1}\right)\right] \cdot \psi\left(a_{1}\right) .
\end{aligned}
$$

Now, suppose that for some $a \neq e$, we have $\psi(a) \neq a$, so that $[\psi(a)]^{-1} a \neq e$, and $d=\phi\left([\psi(a)]^{-1} a\right)$ is defined. It follows that $\phi(d)$ $=[\psi(a)]^{-1} a$, or

$$
\psi(a) \phi(d)=a .
$$

Upon replacing $a_{1}$ by $a$ and $a_{2}$ by $d$ in (3), (4), (5) and (6) and using (7), we obtain

$$
\begin{aligned}
& \phi(a d)=\phi(a) \cdot \phi(a), \\
& \psi(a d)=\psi(a) \cdot \psi(d), \\
& \phi(a d)=\phi(d) \cdot \phi[\psi(d) \cdot \phi(a)], \\
& \psi(a d)=\psi[\psi(d) \phi(a)] \cdot \psi(a) .
\end{aligned}
$$

Comparison of (9) and (11) yields,

$$
\psi(d)=\psi[\psi(d) \cdot \phi(a)]
$$

or

$$
d=\psi(d) \phi(a) .
$$

Replacing $\psi(d) \phi(a)$ by $d$ in (10), we obtain

$$
\phi(a d)=\phi(d) \cdot \phi(d) .
$$

Comparing (8) and (12) we obtain $[\phi(a)]^{2}=[\phi(d)]^{2}$, or $\left(\phi(a)[\phi(d)]^{-1}\right)^{2}$ $=e$.

In Case 1 , we note that $a \neq e$ implies $\psi(a) \neq a$. For otherwise $b a b$ 
$=\phi(a) b a$, from which it follows that $b a=\phi(a) b a b=[\phi(a)]^{2} b a$, or $[\phi(a)]^{2}=e$, which is not possible, since $A$ contains no element of order 2. Thus, we have shown that $a \neq e$ implies the existence of an element $d$ in $A$, such that $\psi(a) \cdot \phi(d)=a$ and $\left(\phi(a)[\phi(d)]^{-1}\right)^{2}=e$. The last equation implies $\phi(a)=\phi(d)$, whence $a=d$, and $\psi(a) \phi(a)=a$. This completes the proof for Case 1.

In Case $2, t_{0}=t$, the unique element of order 2 in $A$. In this case, if $a \neq e$, and $a \neq \phi(t)$ then $\psi(a) \neq a$. Otherwise, we have, as in Case 1, $[\phi(a)]^{2}=e$, which implies $\phi(a)=t$, or $a=\phi(t)$. Thus, there exists a $d \in A$ such that $\psi(a) \cdot \phi(d)=a$ and $\left(\phi(a) \cdot[\phi(d)]^{-1}\right)^{2}=e$. This last equation implies that either $\phi(a)=\phi(d)$ or $\phi(a)=t \phi(d)$. Suppose that $\phi(a)=\phi(d)$; then $a=d$ and we have $\psi(a) \cdot \phi(a)=a$. Consider the two elements of order $2, b$ and $a b a^{-1}$. Since $a \neq e$, they are distinct, and hence by IV, their product $b a b a^{-1}$ displaces all elements of $\mathfrak{M}$. We see, however, that $b a b a^{-1}=\phi(a) b \psi(a) a^{-1}$, and since $\psi(a) \cdot \phi(a)=a$, $\psi(a) a^{-1}=[\phi(a)]^{-1}$, and hence $b a b a^{-1}=\phi(a) b[\phi(a)]^{-1}$ is conjugate to $b$. Since $b$ fixes an element of $\mathfrak{M}$, so do its conjugates. This contradiction implies $\phi(a)=t \phi(d)$. We see, then, that for $a \neq e, a \neq \phi(t)$,

$$
\psi(a) \phi(a)=t a .
$$

If $a=\phi(t)$, then $\psi(a) \cdot \phi(a)=\psi(\phi(t)) \cdot t=[\psi(t)]^{-1} \cdot t$, by (2). Further, by (1) $[\psi(t)]^{-1} \cdot t=\phi\left(t^{-1}\right) \cdot t=\phi(t) \cdot t$. Hence (13) holds for all $a \neq e$. It follows that

$$
\psi(t a) \cdot \phi(t a)=a
$$

for all $a \neq t$. This completes the proof of the lemma.

The next lemma follows readily from the preceding one.

LemmA 4. With $t_{0}$ defined as in Lemma 3, if $a \in A, a \neq t_{0}$ then there exists an $x \in A$ such that $[\psi(x)]^{-1} \cdot x=a$.

Proof. Since $a \neq t_{0}, t_{0} a \neq e$. Let $x=\phi\left(t_{0} a\right)$. By (2) we have $\psi(x)$ $=\psi\left(\phi\left(t_{0} a\right)\right)=\left[\psi\left(t_{0} a\right)\right]^{-1}$, and hence $[\psi(x)]^{-1}=\psi\left(t_{0} a\right)$. Thus, $[\psi(x)]^{-1} \cdot x$ $=\psi\left(t_{0} a\right) \cdot \phi\left(t_{0} a\right)=a$, by Lemma 3 .

Proof of THE THEOREM. A group $G$, satisfying the hypotheses of the theorem is, by the Corollary to Lemma 2, an independent $A B A$ group, with $B$ of order two. As such it can be represented isomorphically as a group of permutations of the right cosets $\{A x\}$ of $A$ in $G$. To see that $G$ is isomorphic to the group of affine transformation on a near-field, it is sufficient, in view of Hall's theorem, to show that there is exactly one element $g \in G$ which maps $A$ into $A b$ and displaces every coset. It is clear that the set of elements of $G$ which map $A$ into $A b$ are all of the form $a b$, where $a$ ranges over $A$. From IV 
it follows that $t_{0} b$ displaces all of the cosets. Suppose then that $a \neq t_{0}$. By Lemma 4 there exists an $x \in A$ such that $[\psi(x)]^{-1} \cdot x=a$. Let $a^{\prime}=x a^{-1}$; then $x=a^{\prime} a$ and we have $\left[\psi\left(a^{\prime} a\right)\right]^{-1} \cdot a^{\prime} a=a$. Hence, $a^{\prime}=\psi\left(a^{\prime} a\right)$. We then see that the coset $A b a^{\prime}$ is fixed by $a b$, thus $\left(A b a^{\prime}\right) a b=A \phi\left(a^{\prime} a\right) b \psi\left(a^{\prime} a\right)=A b a^{\prime}$. That is, of all the elements $a b$, mapping $A$ into $A b$, only $t_{0} b$ displaces all of the cosets. Thus, $G$ is isomorphic to the group of affine transformations on a near-field $(K,+, \cdot)$, in which the multiplicative group $K(\cdot)$ is isomorphic to the subgroup $A$ of $G$, and hence is Abelian. Since a commutative near-field is a field, the proof of the theorem is completed.

\section{REFERENCES}

1. Daniel Gorenstein, A class of Frobenius groups, Canad. J. Math. vol. 11 (1959) pp. 39-47.

2. Marshall Hall, Projective planes, Trans. Amer. Math. Soc. vol. 54 (1943) pp. 229-277. Correction, ibid. vol. 65 (1949) pp. 473-474.

UNIVERSITY OF MisSOURI 